di ${ }^{\prime} I^{m} \cdot 5 .-$ Nov. 8. Sereno fugace nel crepuscolo vespertino. Chiaro di Luna in PQ. Osservazioni difficili a $77^{\circ}$ di distanza zenitale. Fu calcolata, ed applicata alle coordinate, la rifrazione. - Nov. 22, 23,24 . Sereno splendido. Osservazioni difficilissime per essere la cometa molto bassa. Essa fu osservata da $80^{\circ}$ ad $83^{\circ}$ di distanza zenitale. Fu calcolata ed applicata la rifrazione. Il divario un pocolino troppo forte fra le declinazioni della cometa ottenute con due stelle il 24 Novembre fa dubitare che la declinazione di *30 sia difettosa per moto proprio incognito, o per altro. Dopo. quest'ultima data si succedettero in questa nostra regione incessanti burrasche boreali ed australi fino a tutt'oggi, che ancora persistono, e per ciò l'osservazione 24. Novembre rimane definitivamente l'ultima vespertina.

\title{
Vorläufige Aufsuchungsephemeride für den Kometen 1852 IV (Westphal).
}

Die hier wiedergegebene Aufsuchungsephemeride für den Westphalschen Kometen umspannt Frühjahr und Sommer I9 I I und bildet somit so ziemlich eine Fortsetzung der in A. N. I 86. I 7 für den gleichen Zweck gegebenen Positionen. Es fehlt mir leider, weil das Arbeitspensum in meinem Bureau in den letzten zwei Jahren stetig und mehr als doppelt so rasch gewachsen ist, wie der Personalstand, die Zeit, um die Berechnung der Störungen nach dem Jahre I 852 weiter zu führen. Immerhin dürfte es mir möglich sein, diese Arbeit bis zum Ende des Jahres I 9 I I zu erledigen, und vorläufig genügen ja bei der großen Entfernung des Kometen von der Erde die gegebenen Positionen noch vollständig.

Die Helligkeitsverhältnisse sind im kommenden Sommer bereits erheblich günstiger, wie aus der folgenden Tabelle ersichtlich ist.

\begin{tabular}{|c|c|c|c|c|}
\hline & $\begin{array}{l}\text { Umlaufszeit } \\
\text { April I o }\end{array}$ & $\begin{array}{c}60 \text { Jahre } \\
\operatorname{I~I~I~}^{\mathrm{m}} \cdot 4\end{array}$ & $\begin{array}{l}\text { 61 Jahre } \\
12^{\mathrm{m}} \cdot 6\end{array}$ & $\begin{array}{c}\text { 62 Jahre } \\
\text { I } 3^{\mathrm{m}} \cdot 4\end{array}$ \\
\hline & Juni I 9 & 10.7 & I 2.2 & I $3 \cdot \mathbf{I}$ \\
\hline & Aug. 28 & 10.3 & I $2 . \mathrm{I}$ & I 3.1 \\
\hline
\end{tabular}

In der Zwischenzeit ist der Komet nicht nur der Sonne um ein Beträchtliches näher gerückt, sondern er steht überdies ungefähr in Opposition, welch' letzteres auch seine Distanz von der Erde um etwa ${ }_{1}^{1} / 2$ Erdbahnradien verringert. Fügt man den in der Tabelle angegebenen Größen noch etwa zwei Größenklassen hinzu, so folgt, daß die damit für den ungünstigsten Fall einer Umlaufszeit von 62 Jahren $z u$ gewärtigende Helligkeit von ca. ${ }^{\mathrm{I}} 5^{\mathrm{m}}$ vielleicht doch schon im heurigen Sommer zu einer Auffindung führen wird, da der günstigere Ort des Kometen einerseits und die um diese Zeit langen Nächte der südlichen Halbkugel andererseits genügend Zeit zu längeren Expositionen bieten.

Vorläufige Ephemeride des Kometen $185^{2}$ IV (Westphal) für I ${ }^{\text {h }}$ M. Z. Berlin. Igri.o.

\begin{tabular}{|c|c|c|c|c|c|c|c|c|c|c|c|c|c|c|c|c|c|c|c|}
\hline \multirow{2}{*}{\multicolumn{2}{|c|}{ I9II }} & \multicolumn{6}{|c|}{ L'mlaufszeit 60 Jahre } & \multicolumn{6}{|c|}{ Umlaufszeit 6r Jahre } & \multicolumn{6}{|c|}{ Umlaufszeit 62 Jahre } \\
\hline & & & $\alpha$ & & $\delta$ & $\log r$ & $\log \Delta$ & & $x$ & & 8 & $\log r$ & $\log \Delta$ & & $\alpha$ & & $\delta$ & $\log r$ & $\log \Delta$ \\
\hline \multicolumn{2}{|c|}{ April 10.5} & $o^{h}$ & $4 I^{m} \cdot 2$ & $-58^{\circ}$ & $6: 3$ & 0.7346 & $0.735^{2}$ & $19^{\mathrm{h}}$ & I $2^{\mathrm{m}} \cdot 5$ & $-64^{\circ}$ & I $7: 8$ & 0.8647 & $0.853 \mathrm{I}$ & \multicolumn{2}{|c|}{$18^{\mathrm{h}} 12^{\mathrm{m}} \cdot 1$} & \multicolumn{2}{|c|}{$-65^{\circ} 5^{2} \cdot 6$} & 0.9438 & \multirow[t]{2}{*}{0.9294} \\
\hline & 20.5 & & $47 \cdot 7$ & $5^{8}$ & 51.0 & 0.7297 & & & I 2.9 & 65 & 9.8 & 0.8621 & & & 9.2 & 66 & $3^{6.3}$ & $0.942 \mathrm{I}$ & \\
\hline & $3^{\circ} .5$ & & $52 \cdot 5$ & 59 & 44.1 & 0.7247 & 0.7026 & & I I. I & 66 & $3 \cdot 3$ & 0.8594 & $0.833 \mathrm{I}$ & I 8 & 4.2 & 67 & 16.0 & 0.9403 & 0.9146 \\
\hline \multirow[t]{3}{*}{ Mai } & 10.5 & & $55 \cdot 2$ & 60 & 44.9 & 0.7196 & & 19 & 6.7 & 66 & 55.9 & 0.8566 & & 17 & $57 \cdot 1$ & 67 & 53.0 & $0.93^{85}$ & \\
\hline & 20.5 & & 55.6 & $6 r$ & $5^{2.1}$ & 0.7 I 44 & 0.67 I 5 & I 8 & 59.6 & 67 & 45.2 & 0.8539 & 0.8 I $5^{2}$ & & 47.7 & 68 & 21.9 & $0.937^{\circ}$ & 0.9025 \\
\hline & 30.5 & & 53.1 & 63 & 3.6 & 0.7090 & & & 49.8 & 68 & 27.6 & $0.85 \mathrm{II}$ & & & $37 \cdot I$ & 68 & 41.4 & 0.9349 & \\
\hline \multirow[t]{3}{*}{ Juni } & $9 \cdot 5$ & & $47 \cdot 3$ & 64 & I 5.1 & 0.7035 & $0.642 \mathrm{I}$ & & $37 \cdot 5$ & 68 & 59.7 & 0.8483 & 0.8015 & & $25 \cdot 3$ & 68 & 49.6 & $0.9333^{1}$ & 0.8940 \\
\hline & 19.5 & & 38.0 & 65 & 22.9 & 0.6980 & & & 23.4 & 69 & I 8.7 & 0.8454 & & & 13.0 & 68 & 45.8 & 0.93 I 2 & \\
\hline & 29.5 & & 24.7 & 66 & 20.5 & 0.6922 & 0.6200 & 18 & 8.4 & 69 & 22.0 & 0.8425 & 0.7935 & 17 & I. I & 68 & 29.7 & 0.9293 & 0.8907 \\
\hline \multirow[t]{3}{*}{ Juli } & $9 \cdot 5$ & 20 & 8.3 & 67 & I. 4 & 0.6864 & & 17 & 53.6 & 69 & $9 . I$ & 0.8395 & & 16 & 50.5 & 68 & $2 \cdot 3$ & 0.9274 & \\
\hline & 19.5 & 19 & 49.7 & 67 & 19.4 & 0.6803 & 0.6055 & & 40.1 & 68 & $4 \mathrm{r} .2$ & 0.8365 & 0.7916 & & $4 \mathrm{I} .5$ & 67 & 26.2 & 0.9255 & 0.8922 \\
\hline & 29.5 & & 30.7 & 67 & I 1.9 & 0.6742 & & & 29.7 & 68 & I.O & 0.8334 & & & $34 \cdot 5$ & 66 & $4.3 \cdot 4$ & 0.9236 & \\
\hline \multirow[t]{3}{*}{ Aug. } & 8.5 & 19 & I 3.0 & 66 & $3^{8.6}$ & 0.6678 & 0.6004 & & 21.0 & 67 & I 0.8 & $0.83 \circ 3$ & 0.7953 & & 29.7 & 65 & $5^{6.4}$ & 0.9216 & 0.8978 \\
\hline & 18.5 & I 8 & $5^{8.2}$ & 65 & 42.6 & 0.66 I 3 & & & 14.2 & 66 & I $3 \cdot 1$ & 0.8272 & & & 27.1 & 65 & 7.6 & 0.9 × 6 & \\
\hline & 28.5 & & $47 \cdot 3$ & 64 & 29.2 & 0.6546 & 0.6037 & & 10.8 & 65 & I 9.0 & 0.8240 & 0.8028 & & 26.4 & 64 & 19.0 & 0.9176 & 0.9060 \\
\hline Sept. & $7 \cdot 5$ & 18 & 40.7 & -63 & 3.8 & 0.6478 & & I 7 & I 0.7 & -64 & I 2.9 & 0.8207 & & I 6 & 27.7 & -63 & 32.0 & 0.9 I 56 & प्रा \\
\hline
\end{tabular}

Wien, I9 I im Februar.

Adolf Hnatek.

Komet 1907 V. Fräulein S. Rudseewsky, Studentin der höheren Frauenkurse, Moskau, hat die Bearbeitung des Kometen $1907 \mathrm{~V}=1907 \mathrm{e}$ (Mellish) übernommen und bittet um baldige Bekanntgabe noch nicht veröffentlichter Beobachtungen.

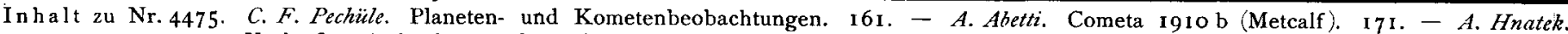
Vorläufige Aufsuchungsephemeride für den Kometen $1852 \mathrm{IV}$ (Westphal). I75. - Komet I9o7 V. I75. 